\title{
The Function of Naringin in Inducing Secretion of Osteoprotegerin and Inhibiting Formation of Osteoclasts
}

\author{
Tong Xu, ${ }^{1,2}$ Lu Wang, ${ }^{1,2}$ You Tao, ${ }^{1,2}$ Yan Ji, ${ }^{1,2}$ Feng Deng, ${ }^{2,3}$ and Xiao-Hong $\mathrm{Wu}^{1,2}$ \\ ${ }^{1}$ Department of Prosthodontics, The Affiliated Hospital of Stomatology, Chongqing Medical University, No. 426 Songshibei Road, \\ Yubei District, Chongqing 401147, China \\ ${ }^{2}$ Chongqing Key Laboratory for Oral Diseases and Biomedical Sciences, No. 426 Songshibei Road, Yubei District, \\ Chongqing 401147, China \\ ${ }^{3}$ Department of Orthodontics, The Affiliated Hospital of Stomatology, Chongqing Medical University, No. 426 Songshibei Road, \\ Yubei District, Chongqing 401147, China
}

Correspondence should be addressed to Xiao-Hong Wu; 104758443@qq.com

Received 16 September 2015; Accepted 24 November 2015

Academic Editor: Jae Youl Cho

Copyright (C) 2016 Tong Xu et al. This is an open access article distributed under the Creative Commons Attribution License, which permits unrestricted use, distribution, and reproduction in any medium, provided the original work is properly cited.

\begin{abstract}
Osteoporosis has become one of the most prevalent and costly diseases in the world. It is a metabolic disease characterized by reduction in bone mass due to an imbalance between bone formation and resorption. Osteoporosis causes fractures, prolongs bone healing, and impedes osseointegration of dental implants. Its pathological features include osteopenia, degradation of bone tissue microstructure, and increase of bone fragility. In traditional Chinese medicine, the herb Rhizoma Drynariae has been commonly used to treat osteoporosis and bone nonunion. However, the precise underlying mechanism is as yet unclear. Osteoprotegerin is a cytokine receptor shown to play an important role in osteoblast differentiation and bone formation. Hence, activators and ligands of osteoprotegerin are promising drug targets and have been the focus of studies on the development of therapeutics against osteoporosis. In the current study, we found that naringin could synergistically enhance the action of $1 \alpha, 25$-dihydroxyvitamin $\mathrm{D}_{3}$ in promoting the secretion of osteoprotegerin by osteoblasts in vitro. In addition, naringin can also influence the generation of osteoclasts and subsequently bone loss during organ culture. In conclusion, this study provides evidence that natural compounds such as naringin have the potential to be used as alternative medicines for the prevention and treatment of osteolysis.
\end{abstract}

\section{Introduction}

The two main cell types responsible for bone remodeling are osteoblasts and osteoclasts. Molecules that can either increase the proliferation of osteoblasts and/or inhibit the differentiation of osteoclasts can contribute to the formation of new bone.

The main cause of osteoporosis is a dysfunction of osteoblasts and/or hyperfunction of osteoclasts. Therefore, therapeutic strategies are aimed at improving the proliferation and differentiation of osteoblasts, accompanied by either inhibiting osteoclastogenesis or facilitating dysfunction of osteoclasts. Statin, an HMG-CoA (3-hydroxy-3-methylglutaryl coenzyme A) reductase inhibitor, is currently being used clinically for the treatment of osteoporosis. Other drugs include bone resorption inhibitors like bisphosphonates and calcitonin, all of which contribute to the maintenance of bone mass by inhibiting the function of osteoclasts. However, the negative side effects of statin necessitate investigations into alternative treatment strategies. Currently, there is considerable interest in identifying new and better drugs that can not only stimulate bone formation but also suppress the differentiation of osteoclasts.

The hormone $\mathrm{VD}_{3}$ is known to play a crucial role in regulating not only calcium homeostasis but also cell growth and differentiation. Osteoblasts are one of the important targets of $\mathrm{VD}_{3} . \mathrm{VD}_{3}$ were shown to stimulate osteoblast activity and promote the release of a variety of osteoclast correlation factors including osteoprotegerin (OPG), RANKL (receptor activator of unclear factor kappa B ligand), MCSF (macrophage colony-stimulating factor), and IL-6 (interleukin-6). In this way, $\mathrm{VD}_{3}$ can control the dynamics of 
TABLE 1: Primer sequences for RT-PCR.

\begin{tabular}{|c|c|c|c|}
\hline Gene & Primer sequence (forward/reverse) & $T$ (annealing) & Cycles \\
\hline OPG & $\begin{array}{l}5^{\prime} \text {-TCCTGGCACCTACCTAAAACAGCA-3' } \\
5^{\prime} \text {-CTACACTCTCGGCATTCACTTTGG-3' }\end{array}$ & 57 & 35 \\
\hline RANKL & $\begin{array}{c}\text { 5'-ATGATGGAAGGCTCATGGTTG-3' }^{\prime} \\
5^{\prime} \text {-TGTTGGCGTACAGGTAATAGAA-3' }\end{array}$ & 60 & 35 \\
\hline M-CSF & $\begin{array}{c}5^{\prime} \text {-AAGCAGTAACTGAGCAACGGG-3' } \\
5^{\prime} \text {-CCCATATTGCGACACCGAA-3' }\end{array}$ & 59 & 30 \\
\hline IL-6 & $\begin{array}{l}5^{\prime} \text {-GAAATGAGAAAAGAGTTGTGC-3' } \\
5^{\prime} \text {-ATTGGAAATTGGGGTAGGAAG-3' }\end{array}$ & 56 & 35 \\
\hline$\beta$-actin & $\begin{array}{l}5^{\prime} \text {-TGGAATCCTGTGGCATCCATGAAAC-3' } \\
5^{\prime} \text {-TAAAACGCAGCTCAGTAACAGTCCG-3' }\end{array}$ & 61 & 30 \\
\hline
\end{tabular}

bone formation and resorption [1-5]. In addition, Motoyuki Uchida et al. reported that $\mathrm{VD}_{3}$ can induce the expression of matrix metalloproteinases in the MC3T3-E1 osteoblast precursor cell line to trigger and regulate bone resorption [6].

Naringin has osteoinductive properties and was shown to greatly improve the integration of autogenous bone grafts in the skull of rabbits [7]. Additional studies established that the molecular mechanism of function of naringin is similar to statin, namely, complete inhibition of mevalonate and the downstream metabolite of HMG-CoA reductase. On the other hand, Mandadi et al. reported that naringin could affect bone quality, by preserving calcium concentration and increasing antioxidant activity. Additionally, animal studies have shown that naringin possesses antiosteoporotic activity and that it can suppress the formation of osteoclasts [8-10]. To our knowledge, the functional relationship between naringin and osteoclasts has not been reported. In the present study, we investigate the effect of naringin on the differentiation of osteoclasts and the secretion of OPG in vitro, specifically in relation to $\mathrm{VD}_{3}$.

\section{Materials and Methods}

\subsection{Animals}

2.1.1. Cell Culture. Murine osteoblastic MC3T3-E1 cells were cultured in $\alpha$-MEM (Hyclone, USA) supplemented with $10 \%$ fetal bovine serum (FBS, Hyclone, USA). Cells were plated at a density of $3 \times 10^{5}$ cells per $100 \mathrm{~mm} 24$ before treatment. Cells were treated with $10^{-7} \mathrm{M} \mathrm{VD}_{3}$ or $10^{-7} \mathrm{M}$ dexamethasone (Dex) (both from Sigma-Aldrich, USA). Naringin (SigmaAldrich, USA), at concentrations of $0,1,10$, and $100 \mathrm{mg} / \mathrm{L}$, was added to the different experimental groups for durations of 72 h, 10 days, 20 days, and 30 days. The medium was changed every three days.

2.2. Bone Organ Culture. In this study, we used four-day old SD rats to prepare bone cultures. Approval was obtained from the local institutional review board and that animal care complied with the guidelines of our institution. All experimental procedures were approved by the Medical Experimental Animal Administrative Committee of Chongqing. The calvaria (or skullcap) was dissected out aseptically from rats immediately after they were sacrificed by cervical dislocation. During dissection, the periosteum and dura mater were left intact on the surface of the skull. Each calvaria was trimmed, divided into four pieces (two parietal, one frontal, and one occipital skull bone), and placed into a 24-well tissue culture plate in $\alpha$-MEM supplemented $10 \%$ FBS, $20 \mathrm{mM}$ HEPES, $10^{-7} \mathrm{M} \mathrm{VD}_{3}, 10^{-7} \mathrm{M} \mathrm{Dex}, 100 \mathrm{U} / \mathrm{mL}$ penicillin, and $100 \mu \mathrm{g} / \mathrm{mL}$ streptomycin. Cultures were incubated at $37^{\circ} \mathrm{C}$ in a humidified atmosphere containing $5 \% \mathrm{CO}_{2}$. Next, we added naringin at concentrations of $0,1,10$, and $100 \mathrm{mg} / \mathrm{L}$ to the test groups after one day of culture, for a duration of $24 \mathrm{~h}, 3$ days, 7 days, and 10 days. The medium was changed every three days. To make comparisons across the different experimental conditions, we used bone tissues extracted from a single litter of mice, unless otherwise indicated by an axis break in the figures.

2.3. Reverse Transcription-Polymerase Chain Reaction (RTPCR). Total RNA was isolated from MC3T3-E1 cells using TRIZOL reagent (Invitrogen, Carlsbad, CA), according to the manufacturer's instructions. The concentration of RNA was determined by measuring the optical density of the sample at $260 \mathrm{~nm}$. Equal amounts of all RNA samples were then reverse-transcribed. These cDNA samples were then analyzed for the expression of markers of osteogenic differentiation, including OPG, RANKL, M-CSF, and IL-6. The housekeeping gene $\beta$-actin was used as the internal control for normalizing gene expression level. Using the cDNA as the template, we next performed gene-specific semiquantitative PCR for OPG, RANKL, M-CSF, IL-6, and $\beta$-actin. Primer sequences and PCR reaction conditions are listed in Table 1 . All RT-PCR products were separated and visualized on $1 \%$ agarose gel containing $0.5 \mathrm{mg} / \mathrm{mL}$ ethidium bromide. To quantify the expression level of the target genes, the DNA bands were first photographed under ultraviolet illumination (Bio-Rad ChemiDoc MP, USA) and then analyzed by densitometry. All PCR reactions generated products in the linear range of amplification, so that band intensity could be directly correlated with the quantity of DNA. Final mRNA levels of all markers were determined after normalizing their densitometry values to $\beta$-actin (Bio-Rad ChemiDoc MP, USA). 


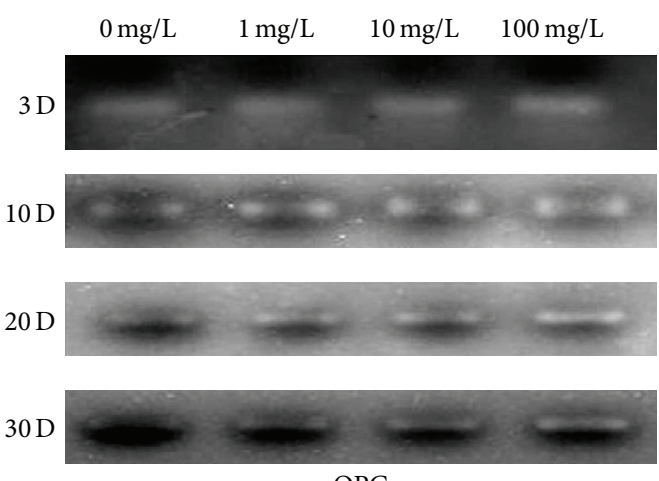

OPG

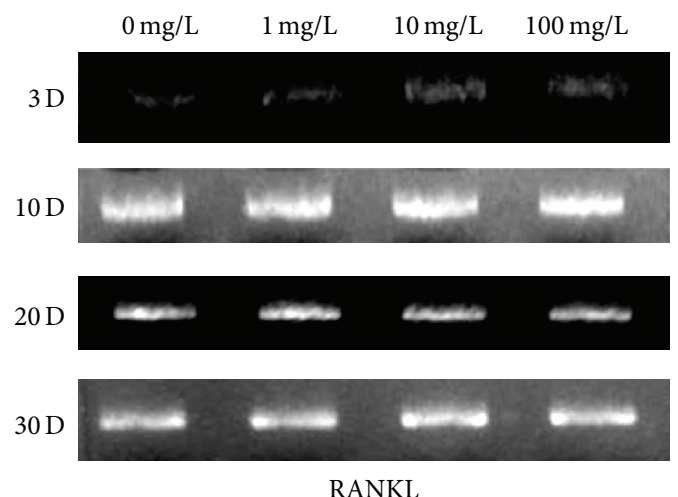

RANKL

FIGURE 1: Analysis of OPG and RANKL expression by RT-PCR. Semiquantitative RT-PCR reactions were performed to estimate the expression level of OPG and RANKL in cells cultured under different experimental conditions ( $0,1,10$, and $100 \mathrm{mg} / \mathrm{L}$ naringin) for 1 to 30 days (D).

2.4. Tartrate-Resistant Acid Phosphatase (TRAP) Staining. Calvarial bone cultures were fixed in 95\% ethanol and 5\% glacial acetic acid for $10 \mathrm{~min}$, then washed with PBS twice, and stained for TRAP activity using a kit (Sigma-Aldrich, USA), according to the manufacturer's instructions. Following staining at $37^{\circ} \mathrm{C}$ in a humid and light-protected incubator for $15 \mathrm{~min}$, the cultures were washed with distilled water three times and observed under the microscope. TRAP-positive cells appeared either red or purple. Each stained calvarial bone culture was scanned completely in a raster fashion by transmitted light microscopy (Nikon Eclipse 80i, Japan) at a final magnification of 200x. Finally, the number of TRAPpositive cells in each sample was counted.

2.5. ELISA-Based Quantification of Secreted OPG. To detect and estimate OPG levels in the culture medium, we performed ELISA (R\&D, USA). Prior to the assay, MC3T3-E1 cells were treated with different concentrations of naringin for 10, 20, and 30 days, while the calvarial bone cultures were treated for $1,3,7$, and 10 days. The culture medium was collected in Eppendorf (EP) tubes and stored at $-80^{\circ} \mathrm{C}$ until ready for analysis. ELISA was performed according to the manufacturer's instructions. The optical density of immunostained cultures was measured using an ELISA reader.

2.6. Measurement of Calcium Concentration in the Bone Culture Medium. To determine the concentration of calcium dissolved from bone slices, the supernatant of calvarial bone cultures treated with different concentrations of naringin was harvested at $1,3,7$, and 10 days. These samples were collected in EP tubes, stored at $-80^{\circ} \mathrm{C}$, and diluted 1:20 with ultrapure water before assay. The total calcium concentration was determined using an atomic absorption spectrophotometer (AAS) (HITACHI Z-5000, Japan).

2.7. Statistical Analyses. Statistical analysis was performed by using the SPSS (SPSS, Chicago, IL, USA) software package. Data from semiquantitative PCR, ELISA, AAS-based $\mathrm{Ca}^{2+}$ measurement, and TRAP activity staining are represented as the mean of three independent repetitions of the assay. Krusklal-Walis $H$ test was used to determine the statistical significance of differences between the test and control groups. $P$ value $<0.05$ was considered statistically significant.

\section{Results}

3.1. Naringin Induces OPG Expression in Osteoblasts. To determine the effect of naringin on the expression of osteoprotegerin, we first treated the osteoblast precursor cell line MC3T3-E1, with different concentrations of naringin for different periods of time. Next, we extracted total RNA from control and treated cultures at different time points and measured the mRNA levels of OPG, RANKL, M-CSF, and IL-6 using semiquantitative RT-PCR. Compared with the control group, we found a significant increase in OPG mRNA expression in naringin-exposed cultures in a time- and dosedependent manner (Figure 1). In contrast, the expression of RANKL, M-CSF, and IL- 6 remained the same in control and experimental groups.

3.2. Naringin Inhibits the Generation of Osteoclasts in a Timeand Concentration-Dependent Manner. We examined the effects of naringin on the composition of calvarial cultures by first staining for TRAP activity and identified a large and significant reduction in the number of TRAP+ osteoclasts (OC) after at least 3 days of exposure (Figure 2).

After $24 \mathrm{~h}$ of culture, we found no difference in the number of TRAP+ OC cells between control and naringintreated groups. However, after calvarial bones were cultured for 3 days, the number of TRAP+ OC cells generated in culture was reduced by $32 \%, 19 \%$, and $26 \%$ in response to $100 \mathrm{mg} / \mathrm{L}, 10 \mathrm{mg} / \mathrm{L}$, and $1 \mathrm{mg} / \mathrm{L}$ naringin, respectively, when compared with control cultures. After 7 days in vitro, we found $67 \%, 53 \%$, and $44 \%$ significant reduction in the quantity of TRAP + OC cells in response to $100 \mathrm{mg} / \mathrm{L}, 10 \mathrm{mg} / \mathrm{L}$, and $1 \mathrm{mg} / \mathrm{L}$ naringin, respectively $(P<0.05)$. Finally, after 10 days in vitro, we found $74 \%, 52 \%$, and $41 \%$ significant reduction in the number of TRAP+ OC cells in response to $100 \mathrm{mg} / \mathrm{L}, 10 \mathrm{mg} / \mathrm{L}$, and $1 \mathrm{mg} / \mathrm{L}$ naringin, respectively $(P<$ $0.01)$. 


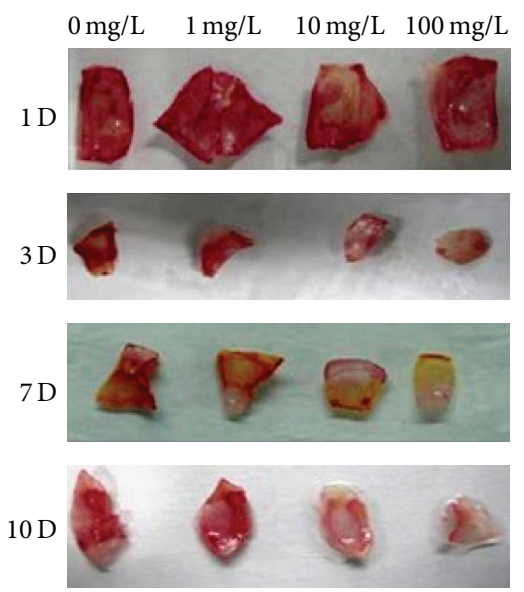

(a)

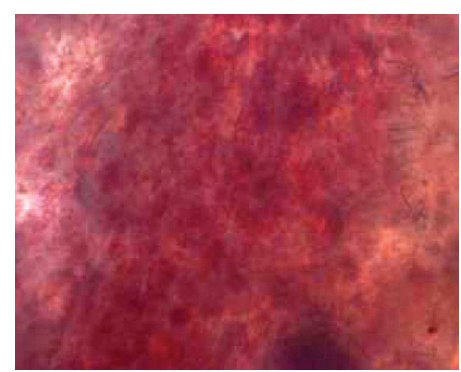

(b)

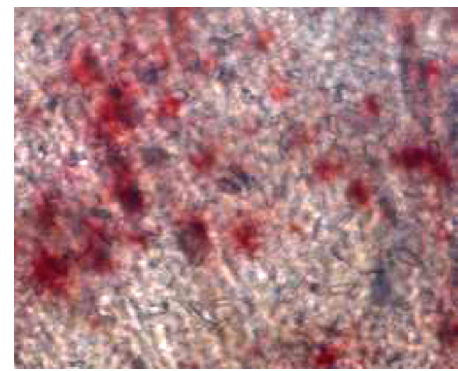

(d)

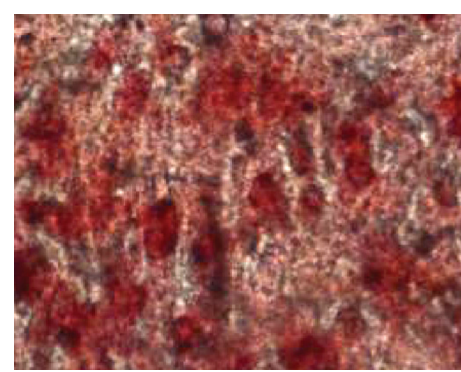

(c)

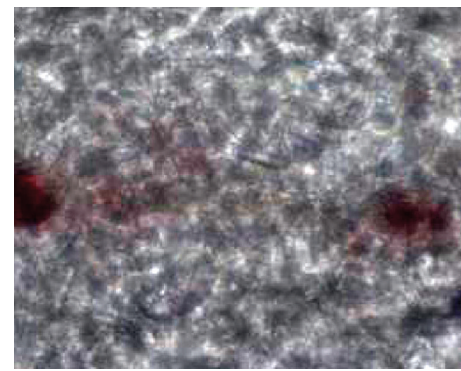

(e)

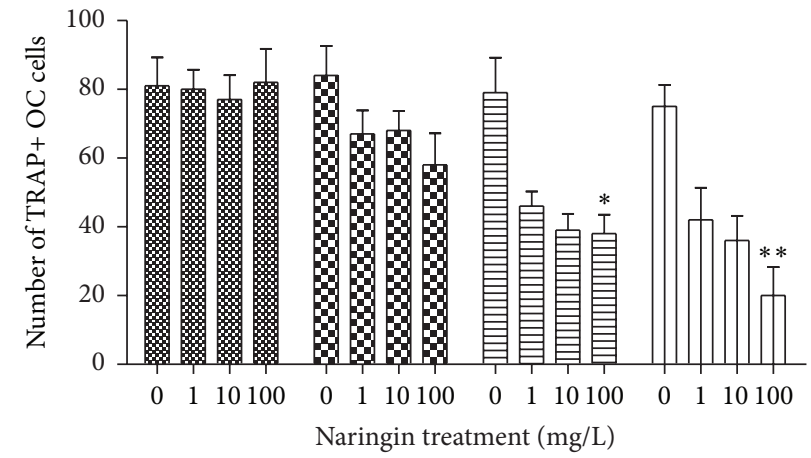

\$2 Day 1

Day 3

$\sqsupseteq$ Day 7

س. Day 10

(f)

FIGURE 2: Effect of naringin on the number of TRAP+ OC cells in calvarial bone cultures. (a) Macroscopic view of TRAP staining in calvarial bone cultures in different experimental conditions $(0,1,10$, and $100 \mathrm{mg} / \mathrm{L}$ naringin), after $1,3,7$, and 10 days (D) in vitro. (b, c, d, and e) Microscopic view of TRAP staining in calvarial bone cultures in different experimental conditions $(0,1,10$, and $100 \mathrm{mg} / \mathrm{L} \mathrm{naringin),} \mathrm{after} 10$ days (D) in vitro. (f) Quantification of the number of TRAP+ OC cells in bone cultures exposed to $0,1,10$, and $100 \mathrm{mg} / \mathrm{L}$ naringin for 1,3 , 7 , and 10 days in vitro. Note the significant reduction in TRAP+ cells at 7 days $\left({ }^{*} P<0.05\right)$ and 10 days $\left({ }^{* *} P<0.01\right)$. 


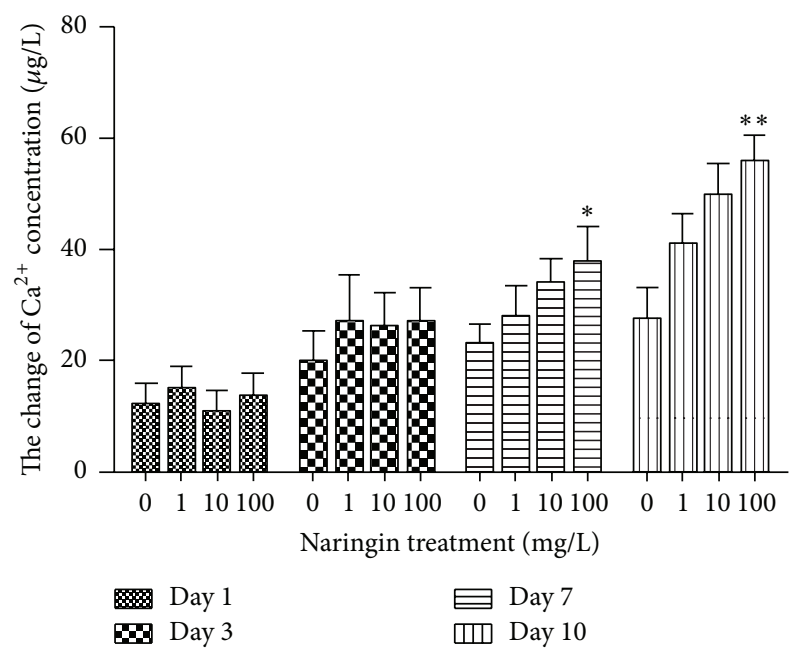

FIgUre 3: Analysis of calcium released into bone culture media. The estimated concentration of dissolved calcium in the supernatant of calvarial bone cultures under different concentrations of naringin $(0$, $1,10$, and $100 \mathrm{mg} / \mathrm{L})$ for $1,3,7\left({ }^{*} P<0.05\right)$, and 10 days $\left({ }^{* *} P<0.01\right)$ in vitro is shown here.

3.3. Naringin Induces Upregulation of OPG in MC3T3-E1 Cells and Calvarial Bone Cultures. To establish whether naringin could influence the secretion of OPG from osteoblast precursors, we assayed for OPG protein levels in the culture supernatant using ELISA. Under control conditions, we detected basal levels of OPG in the culture media of both MC3T3-E1 cells and calvarial bone cultures. However, on exposure to high concentrations of naringin $(100 \mathrm{mg} / \mathrm{L})$, these levels were significantly upregulated.

\subsection{Naringin Exposure Alters Concentration of Dissolved} Calcium in Bone Culture Media. To estimate the effect of naringin on bone formation versus resorption, we quantified the concentration of dissolved calcium ions in culture supernatant under different experimental conditions. The results of AAS- based calcium estimation are shown in Figure 3. We found that after 1 day of exposure to naringin, calcium concentrations were $12 \mu \mathrm{g} / \mathrm{L}, 15 \mu \mathrm{g} / \mathrm{L}, 10 \mu \mathrm{g} / \mathrm{L}$, and $13 \mu \mathrm{g} / \mathrm{L}$ in cultures treated with $100 \mathrm{mg} / \mathrm{L}, 10 \mathrm{mg} / \mathrm{L}, 1 \mathrm{mg} / \mathrm{L}$, and $0 \mathrm{mg} / \mathrm{L}$ naringin, respectively. After 3 days in culture, the concentrations were $27 \mu \mathrm{g} / \mathrm{L}, 26 \mu \mathrm{g} / \mathrm{L}, 27 \mu \mathrm{g} / \mathrm{L}$, and $20 \mu \mathrm{g} / \mathrm{L}$ in response to $100 \mathrm{mg} / \mathrm{L}, 10 \mathrm{mg} / \mathrm{L}, 1 \mathrm{mg} / \mathrm{L}$, and $0 \mathrm{mg} / \mathrm{L}$ naringin, respectively. On day 7 , we detected significant differences in calcium levels, namely, $38 \mu \mathrm{g} / \mathrm{L}, 34 \mu \mathrm{g} / \mathrm{L}, 28 \mu \mathrm{g} / \mathrm{L}$, and $23 \mu \mathrm{g} / \mathrm{L}$ in response to exposure to $100 \mathrm{mg} / \mathrm{L}, 10 \mathrm{mg} / \mathrm{L}, 1 \mathrm{mg} / \mathrm{L}$, and $0 \mathrm{mg} / \mathrm{L}$ naringin, respectively $(P<0.05)$. Finally, after 10 days in vitro, we again found significant differences in calcium concentrations, specifically, $56 \mu \mathrm{g} / \mathrm{L}, 50 \mu \mathrm{g} / \mathrm{L}, 41 \mu \mathrm{g} / \mathrm{L}$, and $28 \mu \mathrm{g} / \mathrm{L}$ in cultures treated with $100 \mathrm{mg} / \mathrm{L}, 10 \mathrm{mg} / \mathrm{L}, 1 \mathrm{mg} / \mathrm{L}$, and $0 \mathrm{mg} / \mathrm{L}$ naringin, respectively $(P<0.01)$.

\section{Discussion}

Osteoporosis is a major, commonly occurring health concern that can result in fractures. Chinese herbal medicines have been used to treat this condition. The compound naringin has been identified as an active component of Rhizoma Drynariae. In recent years, an increasing number of studies have shown that naringin has antioxidant and free radical scavenging properties and that it can protect cells against free oxygen free radical-stimulated $\mathrm{K}^{+}$permeability. In addition, it can induce BMP-2 expression in osteoblasts and enhance osteogenic differentiation and new bone formation [11-15].

Osteoprotegerin was recently described as a member of the TNF receptor superfamily and its cognate ligand, RANKL, has been identified as an important regulator of bone metabolism that mediates paracrine signaling between osteoblasts and osteoclasts [16]. OPG is produced by many different cell types, including bone marrow stromal cells and osteoblasts. It is known to specifically block the fusion and differentiation of osteoclast precursors, and not their proliferation, by binding to RANKL [17]. The physiological function of OPG in regulating bone mass was revealed by studying transgenic mice overexpressing OPG that showed increased skeletal radio density. Significant levels of osteopetrosis and increased bone density observed in these mice were attributed to a decrease in the number of mature osteoclasts. Together with the observation that OPG knockout results in increased bone resorption and skeletal pathologies reminiscent of osteoporosis, the above data establish OPG as a negative regulator of bone resorption in vivo. RANKL is a type II homotrimeric transmembrane protein expressed by cells of the osteoblastic lineage, T lymphocytes, and synoviocytes. It can promote the formation, differentiation, and activation of osteoclasts and lead to enhanced bone resorption. Recent reports have shown that the ratio of OPG to RANKL is an important determinant of bone metabolism and the physiological dynamics between osteoblasts and osteoclasts [18]. In the current study, we have shown that naringin can inhibit the differentiation of osteoclasts by inducing an increase in the OPG/RANKL ratio, resulting from the upregulation of OPG expression rather than downregulation of RANKL.

The hormone $\mathrm{VD}_{3}$ can induce osteoblasts to release a variety of osteoclast correlation factors such as OPG, RANKL, M-CSF, and IL-6. Interestingly, M-CSF and IL-6 are also secreted by osteoblasts and multipotent stem cells and appear to be essential for the proliferation and differentiation of osteoclast progenitors [18-20]. Results from the RT-PCR and ELISA assays presented in this report suggest that exposure to naringin could induce an increase in the activity of $\mathrm{VD}_{3}$ in osteoblast precursor cells leading to a dose-dependent increase in the expression and secretion of the osteoblast differentiation marker OPG.

To the best of my knowledge, this study is the first to investigate the relationship between osteogenic differentiation and naringin activity during bone remodeling in vitro. In contrast to osteoblast precursor cell lines, calvarial bone cultures are composed of all types of cells of the osteoblastic lineage, as well as the bone matrix found inside osseous tissue in vivo. Hence, it is more representative of physiological bone growth and metabolism. In comparison to in vivo experiments, bone organ cultures can be established in a fast, simple, and economical manner and allow an accurate control of the dose and duration of the compounds being 
tested. They also have the added advantage of eliminating variability arising from the extracellular matrix, thus improving the reproducibility of the results obtained. The calvarial bone culture system utilized in this study can be used not only to observe bone formation but also to test the effect of drugs and orthopedic materials on the dynamics of bone tissue growth and function. Therefore, bone organ cultures are a good model to study autocrine and paracrine signaling during bone metabolism [21]. In this study, we show that the highest concentrations of naringin $(100 \mathrm{mg} / \mathrm{L})$ induced that largest decrease in the number of TRAP+ osteoclasts (74\% reduction). These observations are consistent with results from a previous study where TRAP+ OC cells were found to disappear from the endocranial surface of mouse calvarial bones in the presence of naringin. Other studies have shown that naringin can suppress bone resorption induced by positive regulators for osteoclastogenesis including LPS nitric oxide (NO), NO synthase (iNOS), TNFalpha, inducible cyclooxygenase (COX-2), interleukin-6 (IL6), RANKL-induced NF-kappa B, and ERK activation [2226]. Moreover, the reduction in osteoclast differentiation in this study is also consistent with the increase in soluble calcium levels in the organ culture supernatant in the presence of naringin. Altogether, our results suggest that naringin can not only improve bone quality but also reduce the formation or enhance the apoptosis of osteoclasts.

\section{Conclusion}

We show that naringin can increase the activity of $\mathrm{VD}_{3}$ in cultured bone cells and induce them to secrete osteoprotegerin, leading in turn to a reduction in the number of osteoclasts and prevention of bone loss. This corroborates previous evidence of the role of naringin in suppressing bone resorption. We therefore conclude that naringin could be highly beneficial in treating osteoporosis, where it could facilitate bone formation by stimulating increased expression of OPG.

\section{Conflict of Interests}

The authors declare that there is no conflict of interests regarding the publication of this paper.

\section{Acknowledgments}

The study was supported by the National Natural Science Foundation of China (81200767/H1402) and Chongqing Municipal Health Bureau (2012-2-8).

\section{References}

[1] D. D’Ambrosio, M. Cippitelli, M. G. Cocciolo et al., "Inhibition of IL-12 production by 1,25-dihydroxyvitamin D3. Involvement of NF- $\kappa \mathrm{B}$ downregulation in transcriptional repression of the p40 gene," The Journal of Clinical Investigation, vol. 101, no. 1, pp. 252-262, 1998.
[2] S. Kim, M. Yamazaki, L. A. Zella, N. K. Shevde, and J. W. Pike, "Activation of receptor activator of NF- $\kappa$ B ligand gene expression by 1,25-dihydroxyvitamin D3 is mediated through multiple long-range enhancers," Molecular and Cellular Biology, vol. 26, no. 17, pp. 6469-6486, 2006.

[3] Y. Kumei, H. Shimokawa, H. Katano et al., "Microgravity induces prostaglandin $\mathrm{E}_{2}$ and interleukin- 6 production in normal rat osteoblasts: Role in bone demineralization," Journal of Biotechnology, vol. 47, no. 2-3, pp. 313-324, 1996.

[4] K. Sato, Y. Fujii, S. Asano et al., "Recombinant human interleukin 1 alpha and beta stimulate mouse osteoblast-like cells (MC3T3-E1) to produce macrophage-colony stimulating activity and prostaglandin $\mathrm{E}_{2}$," Biochemical and Biophysical Research Communications, vol. 141, no. 1, pp. 285-291, 1986.

[5] T. Yamagishi, E. Otsuka, and H. Hagiwara, "Reciprocal control of expression of mRNAs for osteoclast differentiation factor and OPG in osteogenic stromal cells by genistein: evidence for the involvement of topoisomerase II in osteoclastogenesis," Endocrinology, vol. 142, no. 8, pp. 3632-3637, 2001.

[6] M. Uchida, M. Shima, D. Chikazu et al., "Transcriptional induction of matrix metalloproteinase-13 (collagenase-3) by $1 \alpha, 25-$ dihydroxyvitamin D3 in mouse osteoblastic MC3T3-E1 cells," Journal of Bone and Mineral Research, vol. 16, no. 2, pp. 221-230, 2001.

[7] A. B. M. Rabie, R. W. K. Wong, and U. Hägg, "Composite autogenous bone and demineralized bone matrices used to repair defects in the parietal bone of rabbits," British Journal of Oral and Maxillofacial Surgery, vol. 38, no. 5, pp. 565-570, 2000.

[8] M. Hirata, C. Matsumoto, M. Takita, C. Miyaura, and M. Inada, "Naringin suppresses osteoclast formation and enhances bone mass in mice," Journal of Health Science, vol. 55, no. 3, pp. 463467, 2009.

[9] K. Mandadi, M. Ramirez, G. K. Jayaprakasha et al., "Citrus bioactive compounds improve bone quality and plasma antioxidant activity in orchidectomized rats," Phytomedicine, vol. 16, no. 6-7, pp. 513-520, 2009.

[10] M. Wei, Z. Yang, P. Li, Y. Zhang, and W. C. Sse, "Anti-osteoporosis activity of naringin in the retinoic acid-induced osteoporosis model," American Journal of Chinese Medicine, vol. 35, no. 4, pp. 663-667, 2007.

[11] Y. Chen, R. Zheng, Z. Jia, and Y. Ju, "Flavonoids as superoxide scavengers and antioxidants," Free Radical Biology and Medicine, vol. 9, no. 1, pp. 19-21, 1990.

[12] G. Kroyer, "The antioxidant activity of citrus fruit peels," Zeitschrift für Ernährungswissenschaft, vol. 25, no. 1, pp. 63-69, 1986.

[13] I. Maridonneau-Parini, P. Braquet, and R. P. Garay, "Heterogenous effect of flavonoids on $\mathrm{K}^{+}$loss and lipid peroxidation induced by oxygen-free radicals in human red cells," Pharmacological Research Communications, vol. 18, no. 1, pp. 61-72, 1986.

[14] R. W. K. Wong and A. B. M. Rabie, "Effect of naringin collagen graft on bone formation," Biomaterials, vol. 27, no. 9, pp. 1824$1831,2006$.

[15] X. Zhou, P. Zhang, C. Zhang, and Z. Zhu, "Promotion of bone formation by naringin in a titanium particle-induced diabetic murine calvarial osteolysis model," Journal of Orthopaedic Research, vol. 28, no. 4, pp. 451-456, 2010.

[16] S. Theoleyre, Y. Wittrant, S. K. Tat, Y. Fortun, F. Redini, and D. Heymann, "The molecular triad OPG/RANK/RANKL: involvement in the orchestration of pathophysiological bone remodeling," Cytokine and Growth Factor Reviews, vol. 15, no. 6, pp. 457-475, 2004. 
[17] Y. Wittrant, S. Théoleyre, C. Chipoy et al., "RANKL/RANK/ OPG: new therapeutic targets in bone tumours and associated osteolysis," Biochimica et Biophysica Acta, vol. 1704, no. 2, pp. 49-57, 2004.

[18] M. Pei, L. A. Solchaga, J. Seidel et al., "Bioreactors mediate the effectiveness of tissue engineering scaffolds," The FASEB Journal, vol. 16, no. 12, pp. 1691-1694, 2002.

[19] C. V. Gemmiti and R. E. Guldberg, "Fluid flow increases type II collagen deposition and tensile mechanical properties in bioreactor-grown tissue-engineered cartilage," Tissue Engineering, vol. 12, no. 3, pp. 469-479, 2006.

[20] I. Martin, B. Obradovic, S. Treppo et al., "Modulation of the mechanical properties of tissue engineered cartilage," Biorheology, vol. 37, no. 1-2, pp. 141-147, 2000.

[21] E. Persson and U. H. Lerner, "The neuropeptide VIP potentiates IL- 6 production induced by proinflammatory osteotropic cytokines in calvarial osteoblasts and the osteoblastic cell line MC3T3-E1," Biochemical and Biophysical Research Communications, vol. 335, no. 3, pp. 705-711, 2005.

[22] E. S. M. Ang, X. Yang, H. Chen, Q. Liu, M. H. Zheng, and J. Xu, "Naringin abrogates osteoclastogenesis and bone resorption via the inhibition of RANKL-induced NF- $\kappa$ B and ERK activation," FEBS Letters, vol. 585, no. 17, pp. 2755-2762, 2011.

[23] S.-I. Kanno, A. Shouji, A. Tomizawa et al., "Inhibitory effect of naringin on lipopolysaccharide (LPS)-induced endotoxin shock in mice and nitric oxide production in RAW 264.7 macrophages," Life Sciences, vol. 78, no. 7, pp. 673-681, 2006.

[24] K. Kawaguchi, S. Kikuchi, H. Hasegawa, H. Maruyama, H. Morita, and Y. Kumazawa, "Suppression of lipopolysaccharideinduced tumor necrosis factor-release and liver injury in mice by naringin," European Journal of Pharmacology, vol. 368, no. 2-3, pp. 245-250, 1999.

[25] K. Kawaguchi, S.-I. Kikuchi, R. Hasunuma, H. Maruyama, R. Ryll, and Y. Kumazawa, "Suppression of infection-induced endotoxin shock in mice by a citrus flavanone naringin," Planta Medica, vol. 70, no. 1, pp. 17-22, 2004.

[26] K. T. Steeve, P. Marc, T. Sandrine, H. Dominique, and F. Yannick, "IL-6, RANKL, TNF-alpha/IL-1: interrelations in bone resorption pathophysiology," Cytokine and Growth Factor Reviews, vol. 15, no. 1, pp. 49-60, 2004. 


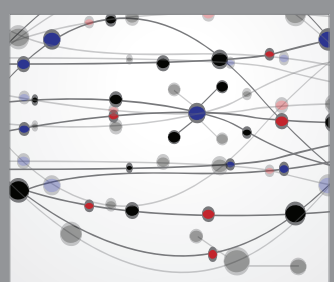

The Scientific World Journal
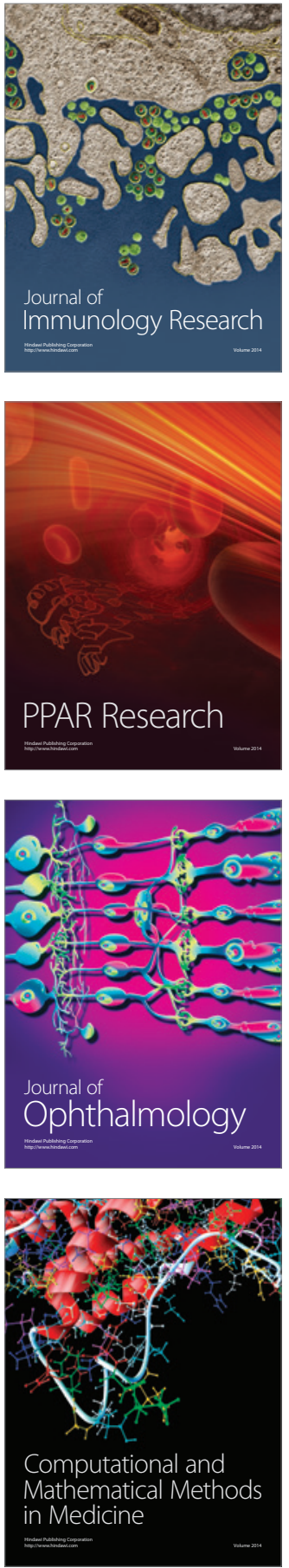

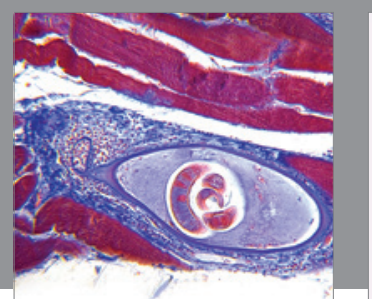

Gastroenterology Research and Practice

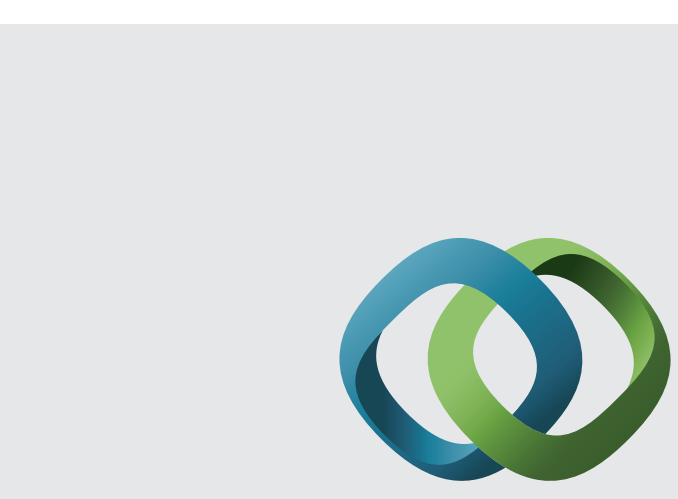

\section{Hindawi}

Submit your manuscripts at

http://www.hindawi.com
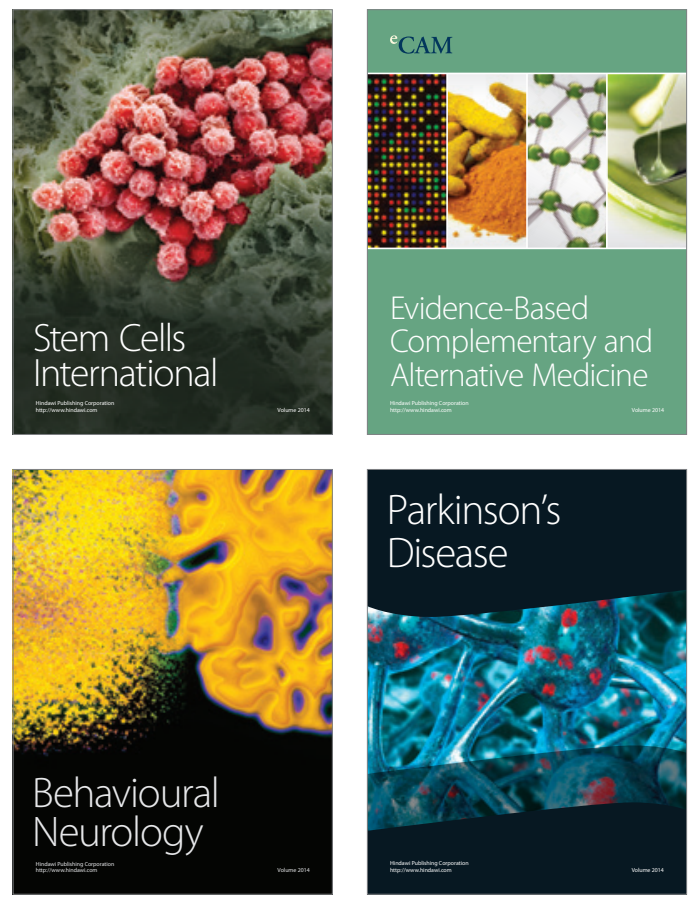
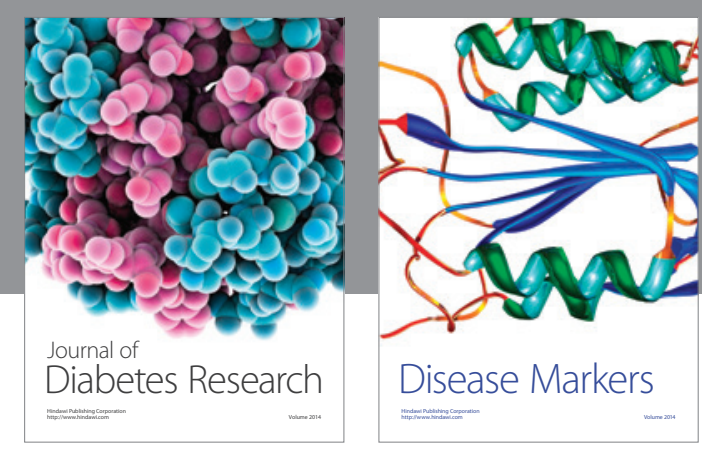

Disease Markers
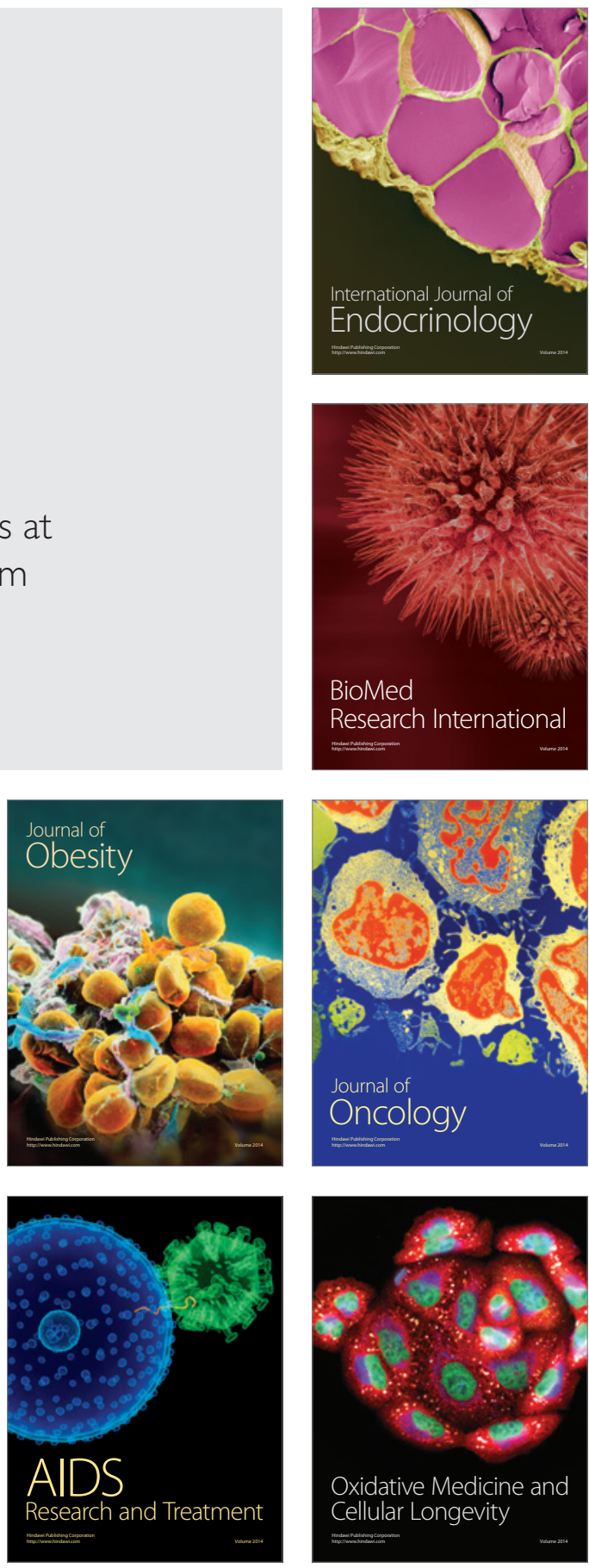